\title{
Fragmentation Metrics in Spectrally-Spatially Flexible Optical Networks
}

\author{
Piotr Lechowicz*, Massimo Tornatore ${ }^{\dagger}$, Adam Włodarczyk* Krzysztof Walkowiak*, \\ ${ }^{*}$ Department of Systems and Computer Networks, Wrocław University of Science and Technology, Poland \\ email: piotr.lechowicz@pwr.edu.pl \\ ${ }^{\dagger}$ Department of Electronics, Information, and Bioengineering, Politecnico di Milano, Italy
}

\begin{abstract}
Spectrally-spatially flexible optical networks (SSFONs) are proposed as a solution for future traffic requirements in optical backbone networks. As SS-FONs operate within flexgrid, the provisioning of lightpaths spanning multiple frequency slots results in spectrum fragmentation, especially in presence of dynamic traffic. Fragmentation, in turn, may lead to blocking of dynamic requests due to the lack of sufficiently-large free spectral windows. In this paper, to reach a better understanding of fragmentation in SS-FON, we extend several metrics used in (singlecore) elastic optical networks to measure the fragmentation in SS-FONs. Next, we apply these metrics to a dynamic-routing algorithm with the goal of minimizing bandwidth blocking. Finally, we analyze the impact of spatial continuity constraint (SCC) on the network fragmentation. Simulations run on two representative network topologies show that the root mean square factor metric yields the best performance in terms of blocking when compared to other analyzed metrics.

Index Terms-spectrally-spatially flexible optical networks; network fragmentation; network optimization; routing, space, and spectrum allocation
\end{abstract}

\section{INTRODUCTION}

According to various statistics, network traffic has been growing in last two decades $30 \%$ to $60 \%$ per year, depending on the nature and penetration of services offered by network operators. Since there is no indication that this growth trend will cease anytime soon, in near future we will face a 'capacity crunch' consisting in incremental exhaustion of available capacity in optical transport networks [1], [2]. One possible solution is space division multiplexing (SDM) - a new optical transmission approach designed to scale the capacity of wavelength division multiplexing (WDM) network and flexgrid elastic optical network (EON) technologies. SDM enables parallel transmissions of a number of co-propagating spatial modes (fibers, cores, or modes) in appropriately designed optical fibers. The concept of spectrally-spatially flexible optical networks (SS-FONs) combines SDM and EONs technologies, and offers several advantages such as substantial increase in transmission capacity, extended flexibility in resource management due to the introduction of spatial domain (spatial modes), and potential cost savings due to the possibility of resource sharing and the use of integrated devices [3]-[6].

One of the potential drawbacks of the flex-grid is the fact that provisioning of lightpaths satisfying continuity and contiguity constraints and using multiple relatively-small (e.g., 12.5-GHz) frequency slices instead of a fixed larger channel spacing (e.g., $50 \mathrm{GHz}$ ) introduces spectrum fragmentation, especially in dynamic traffic context. The key negative consequence of high fragmentation is that dynamic requests can be blocked due to lack of sufficient spectral resources to provision the requests. Therefore, properly addressing the fragmentation problem can allow network operator to allocate more requests in the network. One possible way to mitigate spectrum fragmentation consists in performing periodic or on-demand spectrum defragmentation. However, defragmentation entails complex operational procedure to avoid traffic interruptions, and several studies have instead suggested to to limit fragmentation by performing fragmentation-aware routing, spatial mode, and spectrum allocation (RSSA) [7]-[9].

Several research problems related to fragmentation in EONs have been already investigated (see survey papers [7], [8]). However, only few papers have so far focused on fragmentation in SDM optical networks. In [10], a core classification method is proposed for multi-core fibers (MCFs), where each fiber core is dedicated to realize requests of certain size. A similar approach is presented in [11], where prioritized areas in the frequency domain are used, instead of dedicated spatial mode resources. In [12], a fragmentation-aware routing algorithm with congestion avoidance is proposed. In [13], a crosstalk-aware defragmentation method is described, which uses 'spectrum compactness' metric to trigger and control the defragmentation process. In [9], some of the connections are established using advanced reservation, i.e., these requests can tolerate a certain delay before setup. To the best of our knowledge, even though in the context of EONs several fragmentation metrics have been studied (as external fragmentation, Shannon entropy, access blocking probability [7], [8], and root mean square factor [14], just to name few), there is a lack of investigation on the definition of fragmentation metrics in SS-FONs. Note that in SS-FONs the additional spatial dimension triggers new challenges, since each lightpath can be allocated on various spatial modes. A key issue here is the space continuity constraint (SCC). In particular, if we assume a precise indexing of spatial modes, the same in each network link, the SCC implies that a unique spatial mode shall be assigned to all the channels along a lightpaths. The SCC is enforced by some switching architectures, allowing for devices complexity and cost reduction, at the expense of limited resource allocation flexibility [3], [15]. Since the SCC imposes to assign the same spatial mode index along the whole end-to-end routing path, the RSSA problem becomes 
more challenging and it may significantly increase requests blocking.

The main contribution of this paper is threefold. First, we formulate several fragmentation metrics that can be used in SSFONs. To this end, we adapt fragmentation metrics proposed for EONs to SS-FONs. Second, we discuss simulation results showing performance of the proposed fragmentation metrics applied in dynamic routing in terms of the bandwidth blocking probability. Finally, we investigate the impact of SCC on the spectrum fragmentation in SS-FONs. To the best of our knowledge, this is the first paper that proposes and analyzes various fragmentation metrics in SS-FONs.

The rest of the paper is organized as follows. In Sec. II, we present the considered network model. Sec. III discusses our proposed fragmentation metrics. Finally, in Sec. IV numerical results are presented, and Sec. V concludes this work.

\section{NETWORK MOdEL}

The SS-FON is modeled as directed graph $G(V, E)$ where $V$ is the set of network nodes and $E$ is the set of physical links that interconnects them. Each link $e \in E$ comprises a set of spatial modes $K(e)$, where each spatial mode may refer to fiber in a bundle, core in MCF or mode in multi-mode fiber, without loss of generality. Each spatial mode provides $B$ $\mathrm{THz}$ of available spectrum, divided into $F S$ small frequency slots (slices). By grouping several adjacent slices, a spectral super-channel ( $\mathrm{SCh}$ ) can be formed and used to provision the request. As in [16], the network is equipped with coherent transceivers that use digital signal processing for distortion compensation and operate at fixed baud-rate of 37.5 GBaud. Each transceiver occupies 3 optical carriers of $12.5 \mathrm{GHz}$ width. Table I presents supported bit-rates and transmission reaches for various MFs for single transceiver [16], [17]. If the requested bit-rate exceeds supported maximum capacity of a transceiver, the signal can be transmitted using several adjacent transceivers creating a SCh. Each SCh is separated from the adjacent one with $12.5 \mathrm{GHz}$-wide guardband. Request realization using single SChs allows for spectrum savings, as only one guardband is required. The highest considered request bit-rate is equal to $1 \mathrm{Tbps}$, thus possible $\mathrm{SChs}$ granularities are equal to $3 n+1$, where $n \in\{1, \ldots, 20\}$ [18].

For the sake of simplicity, we do not consider inter-core crosstalk (IC-XT) physical layer impairments, however the applied model can be easily modified to include them, e.g., through an application of worst-case IC-XT estimation [18].

TABLE I: Transmission reach and supported bit-rate for MFs.

\begin{tabular}{l|rrrc}
\hline & BPSK & QPSK & 8-QAM & 16-QAM \\
\hline Transmission reach [km] & 6300 & 3500 & 1200 & 600 \\
Bit-rate [Gbps] & 50 & 100 & 150 & 200 \\
\hline
\end{tabular}

\section{Fragmentation Metrics AND \\ FRAGMENTATION-AWARE DYNAMIC RSSA ALGORITHM}

We consider several metrics adapted from EONs to determine the link fragmentation in SS-FONs, namely, external fragmentation (EF), Shannon entropy (SE), access blocking probability (ABP) [7], root of sum of squares (RSS), root mean square factor (RMSF) [14]. Let $F_{e}^{m}$ denote a fragmentation of link $e \in E$, calculated according to some metric $m$ defined further. The fragmentation of whole network using metric $m$ (denoted as $F_{m}$ ) is calculated as the average value of link fragmentation $F_{e}^{m}$ among all links $e \in E$ as presented in Eq. (1).

$$
F_{m}=\frac{\sum_{e \in E} F_{e}^{m}}{|E|}
$$

It should be stressed that the link fragmentation metrics developed in the context of EONs cannot be directly applied in SS-FONs due to the introduction of the additional spatial dimension. Thus, below we modify the EON link fragmentation metrics in order to address multiple spatial modes. The general idea is to construct the SS-FON metric as the average of the corresponding EON metric calculated for each spatial mode separately.

\section{A. Notation}

Let introduce notation used to define fragmentation metrics. As described in Sec. II, each link $e \in E$ consists of $K(e)$ spatial modes. Each spatial mode $k \in K(e)$ contains $S$ slots. Set $\Gamma(e, k)$ denotes blocks of available contiguous slots on spatial mode $k \in K(e)$ on link $e \in E$, and for each block $\gamma_{e k} \in \Gamma(e, k)$ we define $\left|\gamma_{e k}\right|$ as its size in slices. The highest allocated slice on the spatial mode $k \in K(e)$ on link $e \in E$ is represented by $s_{e k}^{\max }$. Set $G$ denotes types of granularities used by transceivers, i.e., what are the possible sizes of spectral SChs supported by the transceiver [7] assuming guardbands. According to applied network model, the set $G$ contains values equal to $3 n+1$, where $n \in\{1, \ldots, 20\}$. Next, $\lfloor a / b\rfloor$ is the integer division of $a / b$.

\section{B. Fragmentation Metrics}

The first considered metric is EF, originated in computer memory management (Eq. (2)). EF depends on the ratio of largest free contiguous fragment of memory to the total free memory on each core. As the main drawback, the EF metric does not reflect changes in smaller free portions of spectrum until the size of the largest free block is not changing. In consequence, when comparing two fragmented links, the result obtained by this metric is not always meaningful, as it does not prevent from fragmenting smaller portions of spectrum than the largest free block [7], [8].

$$
F_{e}^{E F}=1-\frac{1}{|K(e)|} \cdot \sum_{k \in K(e)} \frac{\max _{\gamma_{e k} \in \Gamma(e, k)}\left|\gamma_{e k}\right|}{\sum_{\gamma_{e k} \in \Gamma(e, k)}\left|\gamma_{e k}\right|}
$$

The SE in information theory is the amount of information available in the message. Eq. (3) presents adaptation for fragmentation in SS-FONs. In contrast to EF, SE takes into the account sizes and amount of smaller portions of free spectrum blocks, which makes it more meaningful to compare two links [7], [8], [19], [20].

$$
F_{e}^{S E}=\frac{1}{|K(e)|} \cdot \sum_{k \in K(e)} \sum_{\gamma_{e k} \in \Gamma(e, k)} \frac{\left|\gamma_{e k}\right|}{S} \cdot \ln \frac{S}{\left|\gamma_{e k}\right|}
$$




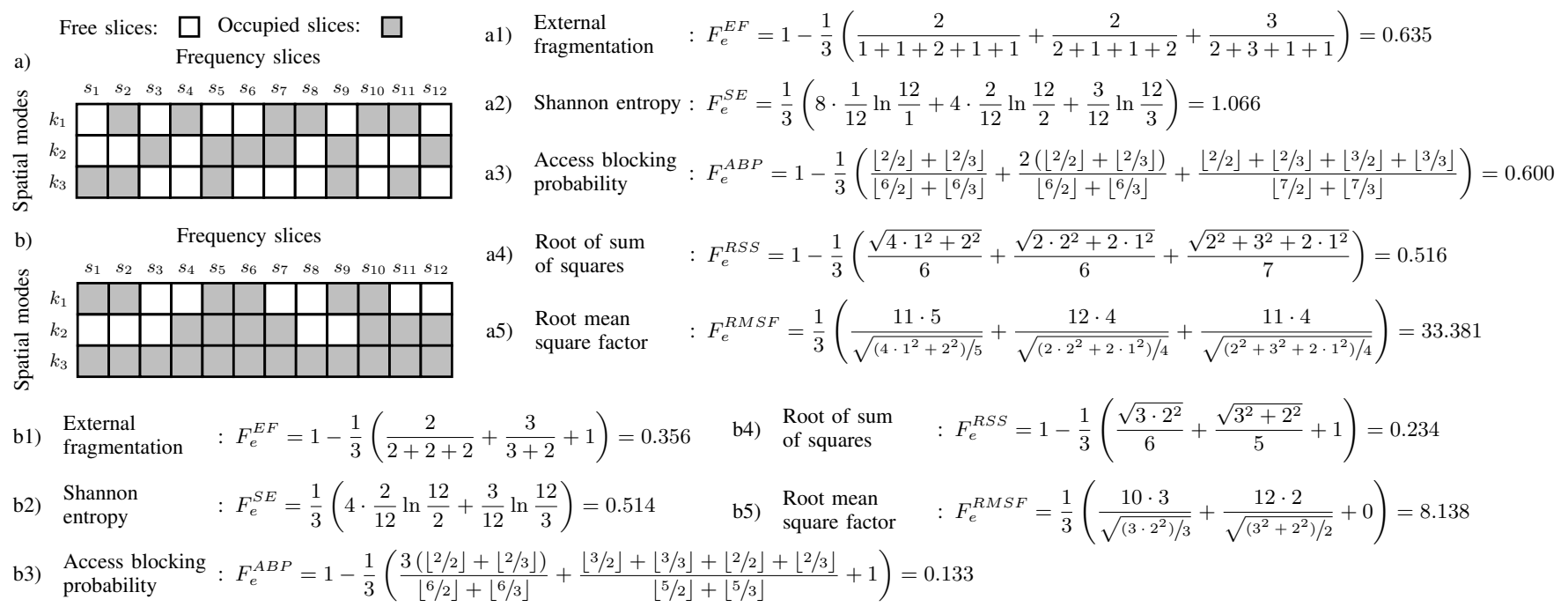

Fig. 1: Example of fragmentation metrics calculations.

The ABP metric is formulated based on the available granularities of the transceivers (Eq. (4)). More specifically, it depends on the ratio between the sum of possible channels created with transceivers operating at various granularities in every free spectrum fragment, to the number of possible channels created with various transceivers granularities assuming that all slots are contiguous. Comparing to other metrics, ABP is aware of the possible sizes of SChs that can be created in the network [7], [8].

$$
F_{e}^{A B P}=1-\frac{1}{|K(e)|} \cdot \sum_{k \in K(e)} \frac{\sum_{\gamma_{e k} \in \Gamma(e, k)} \sum_{g \in G}\left\lfloor\left|\gamma_{e k}\right| / g\right\rfloor}{\sum_{g \in G}\left\lfloor\left(\sum_{\gamma_{e k} \in \Gamma(e, k)}\left|\gamma_{e k}\right|\right) / g\right\rfloor}
$$

Another possible metric is RSS, presented in Eq. (5). It depends on the sum of square roots of sum of sizes of all free segments on each spatial mode. As SE, this metric can reflect the changes in smaller free segments of the spectrum.

$$
F_{e}^{R S S}=1-\frac{1}{|K(e)|} \cdot \sum_{k \in K(e)} \frac{\sqrt{\sum_{\gamma_{e k} \in \Gamma(e, k)}\left(\left|\gamma_{e k}\right|^{2}\right)}}{\sum_{\gamma_{e k} \in \Gamma(e, k)}\left|\gamma_{e k}\right|}
$$

The RMSF is defined in Eq. (6) as a function of the highest allocated slice on each core $S_{e k}^{\max }$, number of free spectrum segments $|\Gamma(e, k)|$ and their sizes $\gamma_{e k} \in \Gamma(e, k)$. The metric increases, when i) a higher slot is allocated in each spatial mode; ii) there are more free contiguous segments on spatial modes, i.e., available slices are divided into a larger set of free segments; iii) the size of large free segments decreases at the cost of increase of smaller segments size [14].

$$
F_{e}^{R M S F}=\frac{1}{|K(e)|} \cdot \sum_{k \in K(e)} \frac{s_{e k}^{\max } \cdot|\Gamma(e, k)|}{\sqrt{\frac{\sum_{\gamma_{e k} \in \Gamma(e, k)}\left|\gamma_{e k}\right|^{2}}{|\Gamma(e, k)|}}}
$$

Note that for metrics EF, ABP and RSS, in order to avoid the division by 0 when there are no available free segments on a spatial mode, the value inside the sum is replaced with 1 .
Contrarily, in RMSF in such case, the value is replaced with 0. Moreover, for all metrics except se and RMSF, values vary within $\langle 0,1\rangle$ range, while for SE and RMSF within $\langle 0,+\infty)$.

In Fig. 1, we present an example of metrics calculation for two different links. The gray squares indicate slices utilized by lightpaths, while white squares are free slices. The operator $\lfloor a / b\rfloor$ denotes the integer division $a / b$. In the example, the available granularities of transceiver are $G=\{2,3\}$, therefore, free segments of size 1 are ignored in the $F_{e}^{A B P}$ equations.

\section{Fragmentation-Aware Dynamic RSSA Algorithm}

In this work, we consider a dynamic routing, i.e., requests arrive dynamically to the network and for each request a lightpath is selected, otherwise the request is rejected. For this purpose, we propose a fragmentation minimizing $k$-shortest path (FM-kSP) heuristic, where the objective is to minimize the amount of rejected traffic. For each request, FM-kSP selects one path out of $k$-shortest paths, in order to realize the lightpath aiming for the minimum increase of the network fragmentation $F_{m}$, considering one of link fragmentation $F_{e}^{m}$ definitions. In more detail, when a new request arrives to the network, the algorithm investigates all precomputed candidate shortest routing paths. For each path, the lowest possible $\mathrm{SCh}$ is found, i.e., a SCh with the lowest ending slice index forming a candidate lightpath. Note, the selection of SCh respects

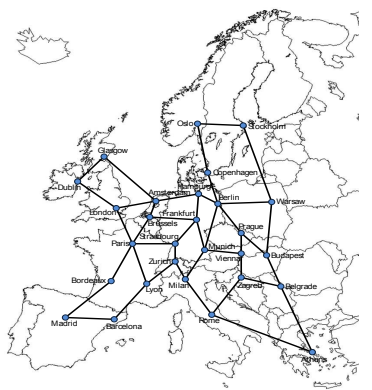

(a) Euro28.

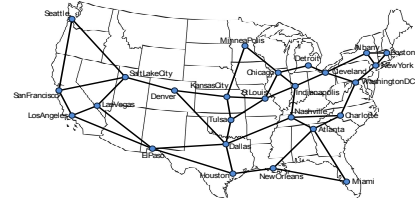

(b) US26.
Fig. 2: Network topologies. 


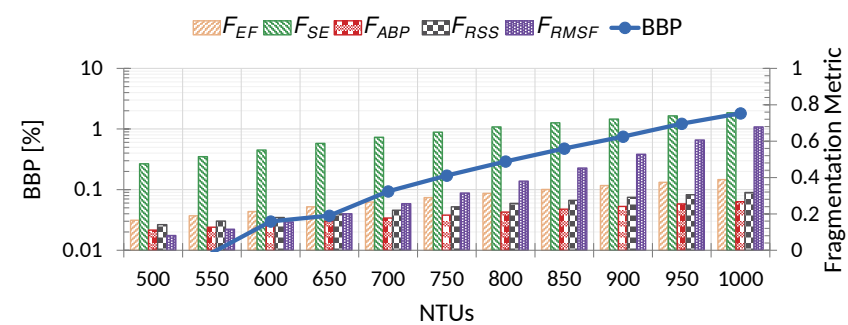

(a) Space continuity constraint relaxed.

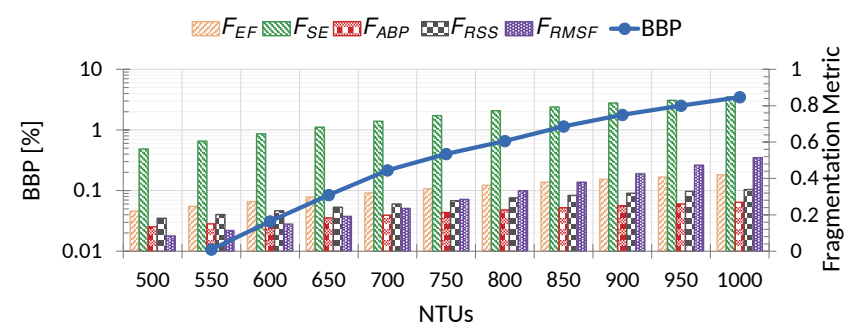

(b) Space continuity constraint.

Fig. 3: BBP and value of fragmentation metrics as a function of NTUs for FF heuristic, Euro28 network topology, traffic A.

the network constraints, i.e., SCC, continuity and contiguity constraints. For each obtained lightpath, the variation in terms of overall network fragmentation $\Delta F$ is calculated as follows:

$$
\Delta F_{m}=\frac{\sum_{e \in E} F_{e}^{m \prime}-F_{e}^{m}}{|E|},
$$

where $F_{e}^{m \prime}$ is the fragmentation measure of link $e$ when the new lightpath is allocated, and $F_{e}^{m}$ is the link fragmentation in current state of network. To calculate $F_{e}^{m}$ and $F_{e}^{m \prime}$, one of the introduced above metrics is used. Next, the lightpath with the lowest fragmentation difference $\Delta F_{m}$ is selected, and request is realized on that lightpath. Note that if the difference is negative, the network fragmentation has improved. If there is no lightpath satisfying all constraints, the request is blocked.

\section{NUMERICAL EXPERIMENTS}

In this section, we evaluate the performance of proposed allocation strategies based on the fragmentation metrics under dynamic traffic requests in SS-FON. We study two network topologies: i) European network (Euro28) - 28 nodes, 82 links and average link length of $625 \mathrm{~km}$; ii) United States network (US26) - 26 nodes, 84 links and average link length of $755 \mathrm{~km}$ (see Fig. 2). The considered SS-FON operates with a flexible grid of $12.5 \mathrm{GHz}$ slices and each network link comprises 7 spatial modes. Each spatial mode provides $4 \mathrm{THz}$ bandwidth divided into 320 slices, each of $12.5 \mathrm{GHz}$ width.

In this study, dynamic traffic requests arrive following a Poisson process with an average arrival rate of $\lambda=10$ requests per unit time. The lifetime of each request follows a negative exponential distribution with an average of $1 / \mu$. Therefore, the traffic load is $\lambda / \mu$ normalized traffic units (NTUs). The requested bit-rate of each request is selected randomly within 50 Gbps to 1 Tbps range, with $50 \mathrm{Gbps}$ granularity. Therefore, traffic of 100 NTUs refers to around $52.5 \mathrm{~Tb} / \mathrm{s}$ of overall traffic load. We investigate two types of requests distributions: i) traffic $\mathrm{A}$ - the source and destination nodes are selected with uniform distribution; ii) traffic B the source and destination nodes are selected proportionally to the distance (in kilometers) between them, i.e., the nodes which are located more closely to each other are more often selected to realize the request. For each analyzed value of traffic load expressed in NTUs, one set with 60000 requests is processed, however, first 10000 requests are excluded from the analysis to enable the network to achieve a steady state. For each request, the number of candidate routing (shortest) paths is 10 . Additionally, we consider two scenarios, with SCC and with SCC relaxed (denoted as no SCC).

The main performance metric is bandwidth blocking probability $(B B P)$, defined as the sum of bit-rates of rejected requests, over the sum of bit-rates of all requests offered to the network. As an additional performance metric, the accepted traffic for $1 \%$ threshold of BBP is used. In more detail, for each scenario the maximum value of traffic load expressed in NTUs is selected, for which the BBP is not larger than $1 \%$, which is a commonly accepted threshold for BBP.

To allocate requests, the FM-kSP algorithm is used (see Sec. III-C) considering all presented fragmentation metrics. As a reference, first-fit $k$-shortest path (FF) method is used, which allocates requests on the first possible $\mathrm{SCh}$, processing candidate paths in increasing order of lengths in $\mathrm{km}$.

The first experiment presents relation between values of various fragmentation metrics and $\mathrm{BBP}$ as a function of NTUs for FF methods, fragmentation metrics are evaluated afterwards traffic allocation. Fig. 3 presents results for traffic A, with and without SCC. The offered traffic load varies from 500 to 1000 NTUs with 50 NTUs granularity. As the values of RMSF (Eq. 6) can be higher than 1, they are normalized to fit the range of other metrics, i.e., from $\langle 0,1\rangle$. Thus, these results are divided by 600 . The first observation is that BBP increases exponentially (linear growth on logarithmic axis) with the linear increase of NTUs offered to the network. Furthermore, the value of fragmentation metrics also increases, suggesting that for higher values of NTUs, the network is more fragmented, and explaining the rapid increase of BBP. When SCC is assumed, the BBP is $120.5 \%$ higher in average between 600 and 1000 NTUs. Note, most of the fragmentation metrics are higher, i.e., $19.4 \%, 14.8 \%, 10.3 \%$, and $16.6 \%$ for EF, SE, ABP and RSS, respectively. Surprisingly, the RMSF is $10.7 \%$ lower assuming SCC. Similar trends were obtained for traffic B and for US26 network topology and are not reported.

In the next experiment, performance of proposed FM-kSP algorithm using various fragmentation metrics is compared in terms of BBP. Fig. 4 presents BBP for various algorithms as a function of NTUs. In the following results, the names of metrics correspond to the FM-kSP algorithm utilizing that metric, exceptd FF which denotes the reference first fit $k$ shortest path algorithm. The first observation is that the RMSF algorithm provides the lowest BBP for most values of NTUs. For instance, for 1000 NTUs the RMSF allows to decrease 


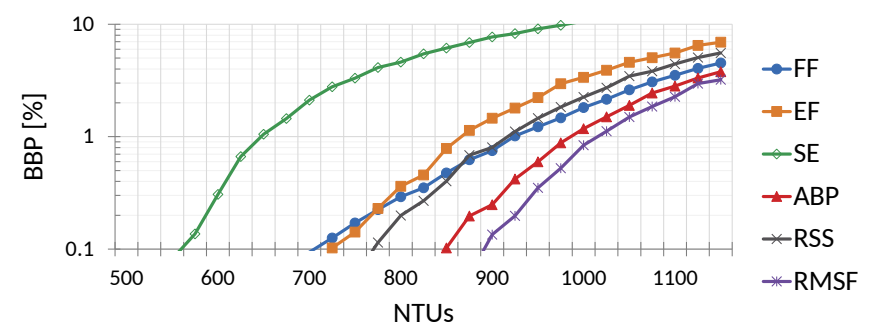

(a) Euro28 network, traffic A, space continuity constraint relaxed.

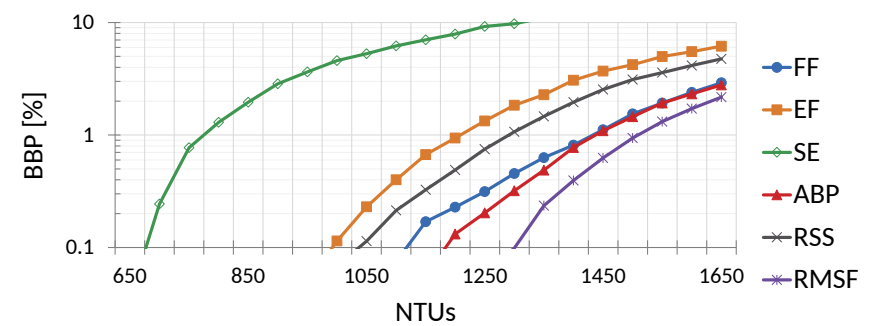

(c) Euro28 network, traffic B, space continuity constraint relaxed.

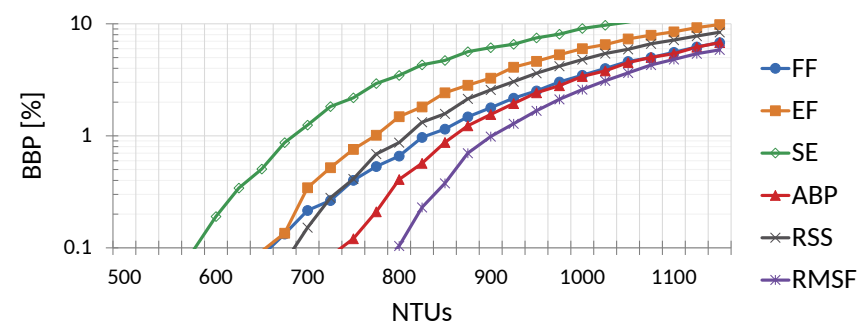

(b) Euro28 network, traffic A, space continuity constraint.

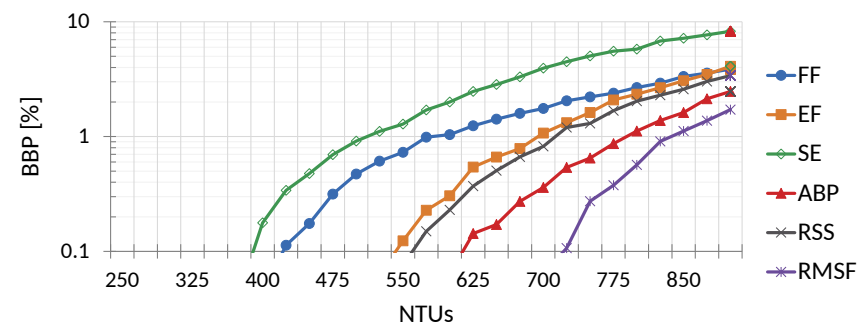

(d) US26 network, traffic A, space continuity constraint relaxed.

Fig. 4: BBP for FM-kSP heuristic using various fragmentation minimizing strategies and FF method as a function of NTUs.

BBP by $28.8 \%$ and $53.8 \%$ in regard to the second best strategy, $\mathrm{ABP}$, and the reference one, FF, respectively, for traffic A and SCC relaxed (Fig. 4a). On average RMSF, allows accepting 5.9 $\%$ and $8.8 \%$ more NTUs for $1 \%$ BBP threshold, in reference to $\mathrm{ABP}$ and $\mathrm{FF}$, respectively. Further, SCC relaxation allows allocating larger NTUs achieving the same BBP, e.g., $11.1 \%$ of gain considering $1 \%$ of BBP and the RMSF strategy in Euro28 network (compare Figs $4 \mathrm{a}$ and $4 \mathrm{~b}$ ). Next, requests following traffic $\mathrm{B}$ provide lower BBP when compared to traffic A, e.g., with a difference of $50.0 \%$ for BBP of $1 \%$ for RMSF (compare Figs. 4a and 4c). Finally, the US26 network can accommodate smaller overall traffic with the same performance than Euro28 network. For BBP of $1 \%$ and RMSF, the difference between allocated NTUs is equal to 21.2\% (compare Figs 4a and 4d). Comparing RMSF to other metrics, the conclusions are as follows: i) as all other metrics, it is aware of the number of free segments; ii) as all other metrics except EF, it is aware of the size of free segments; iii) contrarily to other metrics, it is aware of the highest allocated slice on each spatial mode. The last property might be the main factor determining the best performance of the RMSF metric. In context of EONs, RMSF outperforms two other metrics, namely, spectrum compactness and utilization entropy [14]. However, to the best of our knowledge, RMSF has not been compared to metrics proposed in this paper. Moreover, as EON is a special case of SS-FON assuming one spatial mode, the difference in performance of proposed metrics should be valid also for EON. Next, we provide a detailed analysis of performance differences between considered scenarios for the RMSF metric, which - as shown above - provides the best results in terms of BPP. Fig. 5 presents percentage of served requests as a function of length of selected routing path in hops for Euro28 network for accepted traffic for $1 \%$ threshold of BBP. The results are presented for two numbers of available candidate paths, i.e., $|P|=\{1,10\}$. Fig. 5a presents results for traffic A with SCC and with SCC relaxed, while
Fig. $5 b$ for traffic B. Additionally, the corresponding values of traffic in NTUs are reported. Note, the lines for SCC and SCC relaxed for $|P|=1$ are almost overlapping. Firstly, it is easy to notice that in traffic A the most often selected paths contains 5 hops $(|P|=10)$, while in traffic B the corresponding value is 1 hop, which is strictly related to the source-destination nodes selection probability distribution in each traffic type. Secondly, increasing the number of candidate routing paths, results with selection of longer routing paths, and in consequence, it is possible to accommodate higher amount of NTUs. Additionally, for $|P|=10$, the accepted traffic is higher for SCC relaxed when compared to SCC nonrelaxed. This is because, algorithm in the SCC scenario tends to select longer paths more often. Moreover, relaxation of SCC facilitates allocation of requests even in a fragmented network, since it is possible to freely switch lightpaths among the spatial modes. Contrarily, when $|P|=1$, there is no significant difference between SCC and SCC relaxed case. Finally, the higher values of traffic can be accepted in traffic B comparing to traffic A, as the distances between source-destination nodes in traffic B are more often shorter. Requests with shorter distances are easier to realize, especially in highly fragmented network, as the continuity constraint is more challenging on path involving higher amount of links.

The last Fig. 6 presents percentage of MFs usage for the RMSF strategy for 1\% BBP threshold. For both network topologies, more efficient MFs are more often selected comparing traffic $\mathrm{B}$ to traffic A because average length of shortest path for requests is lower in traffic B. Moreover, when SCC is present, higher amount of more efficient MFs is selected, but achieving least amount of accepted traffic for 1\% BBP threshold. When SCC is relaxed, increased flexibility allows to allocate additional traffic using longer paths. Finally, in US26 less efficient MFs are more often selected, regardless of traffic profile or SCC. The reason for this is that US26 network has higher average link length when compared to Euro28 network. 


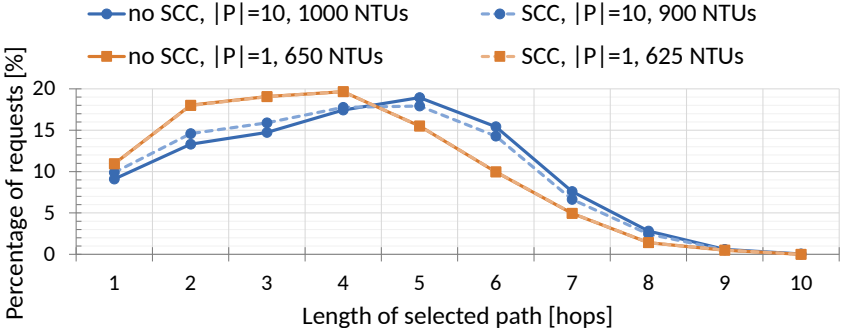

(a) Traffic A.

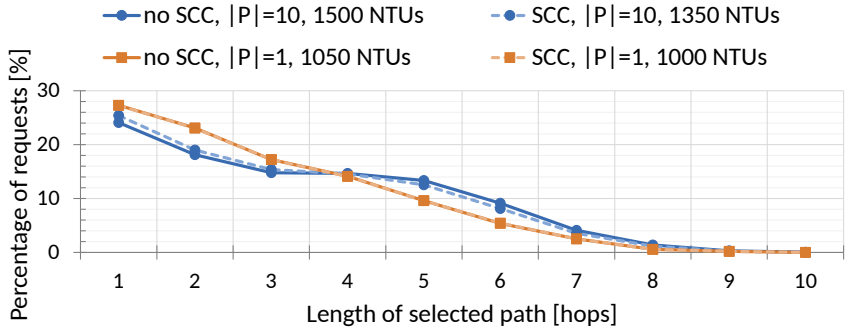

(b) Traffic B.

Fig. 5: Percentage of requests as a function of selected path length for Euro28 network, $1 \%$ threshold of BBP, RMSF strategy.

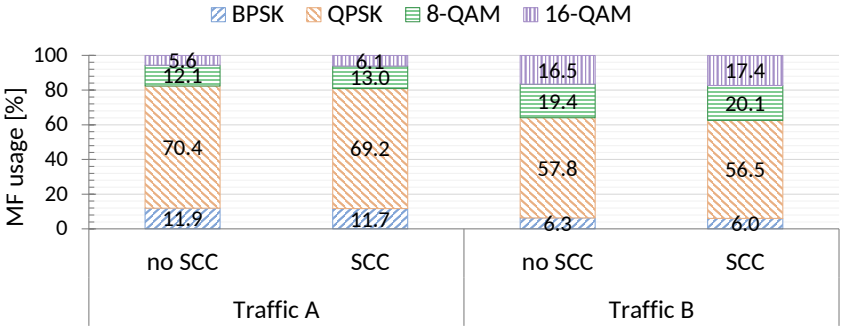

(a) Euro28 network topology

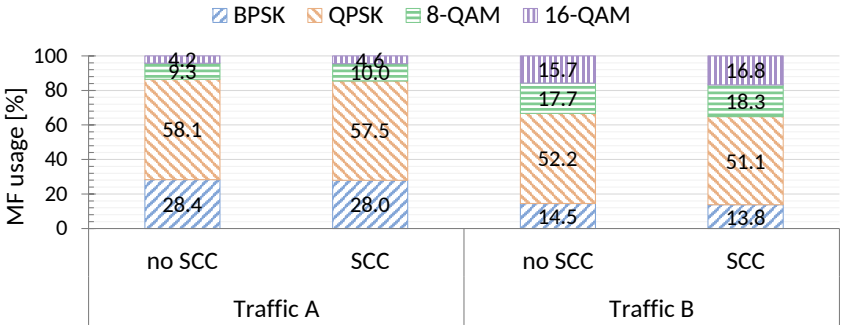

(b) US26 network topology

Fig. 6: MFs percentage usage for 1\% BBP threshold as a function of traffic profile and SCC presence for RMSF strategy.

\section{CONCLUding REMARKS}

In this work, we have focused on the performance of dynamic allocation algorithm applying various fragmentation metrics used to provision dynamic traffic in SS-FONs. According to results of simulations, the RMSF metric allows to decrease BBP and in consequence allows to accept around $8.8 \%$ more traffic (NTUs) assuming $1 \%$ threshold of BBP, when compared to the reference method. Additionally, the relaxation of space continuity constraint allows to allocate $11.1 \%$ more traffic comparing to the case without relaxation. Finally, the traffic profile (i.e., source-destination node selection probability distribution) and the number of available candidate paths have a significant impact on BBP. In the future work, we plan to develop defragmentation methods in SSFONs based on proposed fragmentation metrics.

\section{ACKNOWLEDGMENTS}

The work of P. Lechowicz, K. Walkowiak was supported by National Science Centre, Poland under Grant 2015/19/B/ST7/02490 and statutory funds of Dep. of Systems and Computer Networks, Wroclaw Univ. of Sci. and Techn.

\section{REFERENCES}

[1] P. J. Winzer, "Optical networking beyond wdm," IEEE Photonics Journal, vol. 4, no. 2, pp. 647-651, 2012.

[2] — "Spatial multiplexing in fiber optics: The 10x scaling of metro/core capacities," Bell Labs Techn. J., vol. 19, p. 22-30, 2014.

[3] D. M. Marom and M. Blau, "Switching solutions for WDM-SDM optical networks," IEEE Comm. Mag., vol. 53, no. 2, pp. 60-68, 2015.

[4] G. M. Saridis, D. Alexandropoulos et al., "Survey and evaluation of space division multiplexing: From technologies to optical networks," IEEE Commun. Surv. Tut., vol. 17, no. 4, pp. 2136-2156, 2015.

[5] B. Shariati et al., "Realizing spectrally-spatially flexible optical networks," IEEE Photon. Society Newsletter, vol. 31, no. 6, pp. 4-9, 2017.

[6] M. Klinkowski, P. Lechowicz, and K. Walkowiak, "Survey of resource allocation schemes and algorithms in spectrally-spatially flexible optical networking," Opt. Switch. Netw., vol. 27, pp. 58-78, 2018.
[7] B. C. Chatterjee, S. Ba, and E. Oki, "Fragmentation problems and management approaches in elastic optical networks: A survey," IEEE Commun. Surv. Tut., vol. 20, no. 1, pp. 183-210, 2018.

[8] D. Amar, E. Le Rouzic et al., "Spectrum fragmentation issue in flexible optical networks: analysis and good practices," Photonic Netw. Commun., vol. 29, no. 3, pp. 230-243, 2015.

[9] S. Sugihara et al., "Dynamic resource allocation for immediate and advance reservation in space-division-multiplexing-based elastic optical networks," IEEE/OSA J. Opt. Commun. Netw., vol. 9, no. 3, pp. 183197, 2017.

[10] S. Fujii, Y. Hirota et al., "On-demand spectrum and core allocation for reducing crosstalk in multicore fibers in elastic optical networks," IEEE/OSA J. Opt. Commun. Netw., vol. 6, no. 12, pp. 1059-1071, 2014.

[11] H. Tode and Y. Hirota, "Routing, spectrum, and core and/or mode assignment on space-division multiplexing optical networks [invited]," IEEE/OSA J. Opt. Commun. Netw., vol. 9, no. 1, pp. A99-A113, 2017.

[12] L. Liu et al., "3d elastic optical networks in temporal, spectral, and spatial domains with fragmentation-aware rssma algorithms," in The European Conf. on Optical Communication (ECOC), 2014, pp. 1-3.

[13] Y. Zhao et al., "Crosstalk-aware spectrum defragmentation based on spectrum compactness in space division multiplexing enabled elastic optical networks with multicore fiber," IEEE Access, vol. 6, pp. $15346-$ 15355,2018

[14] Z. Ye, A. N. Patel et al., "Root mean square (rms) factor for assessing spectral fragmentation in flexible grid optical networks," in 2014 OptoElectronics and Communication Conference and Australian Conference on Optical Fibre Technology, 2014, pp. 357-358.

[15] R. Rumipamba-Zambrano et al., "Space continuity constraint in dynamic flex-grid/sdm optical core networks: An evaluation with spatial and spectral super-channels," Comput. Commun., vol. 126, pp. 38-49, 2018.

[16] C. Rottondi, M. Tornatore, and G. Gavioli, "Optical ring metro networks with flexible grid and distance-adaptive optical coherent transceivers," Bell Labs Techn. J., vol. 18, no. 3, pp. 95-110, 2013.

[17] P. S. Khodashenas, J. M. Rivas-Moscoso et al., "Comparison of spectral and spatial super-channel allocation schemes for sdm networks," J. Light. Techn., vol. 34, no. 11, pp. 2710-2716, 2016.

[18] K. Walkowiak et al., "Dynamic routing in spectrally spatially flexible optical networks with back-to-back regeneration," IEEE/OSA J. Opt. Commun. Netw., vol. 10, no. 5, pp. 523-534, 2018.

[19] A. Horota et al., "Routing and spectrum assignment algorithm with most fragmented path first in elastic optical networks," in 7th IEEE LatinAmerican Conf. on Communications (LATINCOM), 2015, pp. 1-6.

[20] J. Shen, J. Chen, and Y. Sun, "Fragmentation aware routing and spectrum assignment algorithm for elastic optical networks," in TENCON 2015 . 2015 IEEE Region 10 Conference, 2015, pp. 1-4. 